\title{
Cellular and molecular aspects of iron and immune function
}

\author{
Jeremy H. Brock ${ }^{1 *}$ and Victoriano Mulero ${ }^{2}$ \\ ${ }^{1}$ Department of Immunology, Western Infirmary, Glasgow G11 6NT, UK \\ ${ }^{2}$ Facultad de Biología, Universidad de Murcia, Murcia, Spain
}

\begin{abstract}
Fe plays a critical role in the immune system and defence against infection. However, many aspects of the way in which Fe influences these processes at the molecular and cellular level are unclear. The present review summarizes the role of $\mathrm{Fe}$ in lymphocyte activation and proliferation, and discusses how $\mathrm{Fe}$ is handled by macrophages.
\end{abstract}

Iron: Lymphocyte activation and proliferation: Macrophages

Fe plays a critical role in many metabolic processes. While its major function is $\mathrm{O}_{2}$ transport by haemoglobin, it is also a crucial cofactor in many other enzymes, whose function may be reduced if there is an inadequate supply of Fe. The immune system consists of a complex network of cells and molecules whose activities may be up regulated in highly specific ways following antigenic challenge, and it is therefore not surprising that abnormal Fe status can lead to impaired immune function.

The whole area of $\mathrm{Fe}$ and its relationship with immunity is complex, and has been extensively reviewed (Brock, 1993; Jurado, 1997; Walter et al. 1997). Moreover, many cells and molecules participate in immune reactions. Two of the key cells involved are lymphocytes and macrophages, and the present review will therefore be limited to their function in relation to Fe status, in particular to studies by the authors related to how Fe affects lymphocyte activation and proliferation, and how macrophages handle Fe.

\section{Iron and lymphocyte function}

It has been known for many years that the proliferative phase of lymphocyte activation is an Fe-requiring step, mainly because $\mathrm{Fe}$ is essential for enzymes such as ribonuleotide reductase, involved in DNA synthesis (Leberman et al. 1984). This finding corroborates a large number of clinical studies, reviewed previously (Brock, 1993), which have found reduced $\mathrm{T}$-cell function in vivo, as manifested by impaired skin-test reactions, and reduced in vitro proliferation of T-cells from Fe-deficient individuals. Activated lymphocytes, like other Fe-requiring cells, normally acquire Fe from transferrin by a mechanism involving interaction with cell-surface transferrin receptors, internalization of the complex and release of $\mathrm{Fe}$ in an acidic endosomal compartment (Baker \& Morgan, 1994). Uptake of Fe is regulated according to cellular needs by modulating transferrin receptor mRNA stability through the binding of Fe-regulatory proteins (IRP) to the $3^{\prime}$-untranslated region (Haile, 1999). However, although Fe deficiency can up regulate transferrin receptor expression, it can also reduce the $\mathrm{Fe}$ saturation of transferrin to a level where it can no longer provide optimal Fe delivery, and as a consequence proliferation is impaired (Mainou-Fowler \& Brock, 1985). On the other hand, Fe overload can lead to complete saturation of transferrin and the presence of non-transferrinbound $\mathrm{Fe}$ in plasma, which can also inhibit lymphocyte proliferation (Djeha \& Brock, 1992).

Although cell proliferation is the most clearly established step in lymphocyte activation in which Fe plays a crucial role, other aspects of lymphocyte function may also be susceptible to modulation by Fe. For example, Fe deficiency in mice reduced protein kinase $\mathrm{C}$ (PKC) activity and translocation in T-cells (Kuvibidila et al. 1999). This finding accords with earlier work showing that $\mathrm{Fe}$-transferrin up regulates expression of PKC- $\beta$ (but not PKC- $\alpha$ ) in various cell lines (Alcantara et al. 1994). Interestingly, nontransferrin-bound Fe could not induce PKC (Alcantara et al. 1991). Further evidence of a role for $\mathrm{Fe}$ in lymphocyte signalling pathways comes from a study showing that phosphatidyl inositol-4,5-biphosphate hydrolysis is reduced in activated spleen cells from Fe-deficient mice (Kuvibidila et al. 1997). However, the involvement of Fe-transferrin in lymphocyte signalling pathways may depend in part on ligand binding to the transferrin receptor rather than $\mathrm{Fe}$ delivery, as the transferrin receptor can associate with the $\mathrm{T}$-cell receptor $\zeta$ chain, and tyrosine phosphorylation can be 
induced using antibodies to the transferrin receptor as well as by transferrin itself (Salmerón et al. 1995). Another $\mathrm{Fe}$-sensitive step in which $\mathrm{Fe}$-transferrin may play a role is in lymphocyte maturation. Blockage of transferrin-bound Fe uptake by thymocytes prevented their maturation into $\alpha \beta$-T-cells, but not into $\gamma \delta$-T-cells (Brekelmans et al. 1994).

In addition, while it seems likely that non-transferrinbound $\mathrm{Fe}$ in the form of citrate or other chelates cannot support lymphocyte activation, there may be other forms, apart from Fe-transferrin, that can do so. Lymphocytes may be able to use intracellularly stored Fe (Golding \& Young, 1995). In addition, haem-haemopexin could substitute for Fe-transferrin in permitting proliferation of lymphoblastoid T-cells, with PKC activation again being implicated as a possible mechanism (Smith et al. 1997). It is suggested that this mechanism of Fe uptake might be important at sites of injury or inflammation.

\section{Iron and macrophages}

Macrophages play a key role in Fe metabolism. They are responsible for catabolism of effete erythrocytes taken up by the liver and spleen, and the release of Fe to the circulation for subsequent binding by transferrin. The $\mathrm{Fe}$ is then transported to the erythroid marrow, where it is reincorporated into haemoglobin in erythroid precursors. Despite this key role in Fe metabolism, the way in which macrophages handle Fe remains the least-understood step in Fe recirculation. Furthermore, the macrophage may hold the key to understanding some disorders of $\mathrm{Fe}$ metabolism, such as the anaemia of chronic disease.

Although most cells acquire Fe for metabolic use from transferrin via the transferrin-receptor endocytotic route, macrophages involved in erythrocyte catabolism take up large amounts of $\mathrm{Fe}$ via phagocytosis of effete erythrocytes. This process, while still incompletely understood, is clearly not regulated by cellular $\mathrm{Fe}$ status in the same way as uptake from transferrin. Moreover, the intracellular routing of $\mathrm{Fe}$ is likely to be different; $\mathrm{Fe}$ acquired from transferrin is released from the carrier molecule (i.e. transferrin) in the early endosome, from which it is transported, probably via Nramp2/DCT1, to the cytoplasm and thence to the mitochondria for metabolic use (Fleming \& Andrews, 1998). In contrast effete erythrocytes are broken down in a phagolysosome, where $\mathrm{Fe}$ must first be released from haemoglobin by haem oxygenase (Kleber et al. 1978). Subsequent steps are unclear, although we have previously shown that $\mathrm{Fe}$ acquired from transferrin and $\mathrm{Fe}$ taken up into phagosomes appear to follow different pathways within the cell (Oria et al. 1988).

In our laboratory we have investigated the effect of various mediators on Fe handling by macrophages, in order to gain some insight into how macrophages handle Fe and how this process is modified during inflammatory disease.

For this purpose, we devised a method of loading the cells with radiolabelled transferrin-antitransferrin immune complexes (Esparza \& Brock, 1981). This method has the advantage of delivering $\mathrm{Fe}$ to the macrophage by a phagocytic route, while at the same time allowing high specific activity of radiolabelling (impossible with labelled erythrocytes) and bypassing the need for haem oxygenase to liberate Fe from its carrier. Using this model we found that inflammatory macrophages released $\mathrm{Fe}$ more slowly than resting macrophages, thus supporting early in vivo work which pointed to a 'reticulo-endothelial blockade' of $\mathrm{Fe}$ recirculation in inflammation (Freireich et al. 1957; Konijn \& Hershko, 1977).

We also investigated whether a similar effect could be induced by cytokines implicated in the inflammatory response. We found that tumour necrosis factor $\alpha$ (TNF- $\alpha$ ), but not interleukin $1 \alpha$ or lipopolysaccharide (LPS), when added to mouse peritoneal macrophages that had ingested $\mathrm{Fe}$-transferrin-antitransferrin immune complexes, reduced Fe release (Alvarez-Hernández et al. 1989). Injection of TNF- $\alpha$ into mice reduced serum Fe, and peritoneal macrophages from these animals incubated with $\mathrm{Fe}$-transferrinantitransferrin immune complexes showed increased uptake and degradation of the complexes without a corresponding increase in Fe release, which would have the overall effect of increasing $\mathrm{Fe}$ retention. Interleukin $1 \alpha$ had no significant effect on serum $\mathrm{Fe}$, and while it also increased the rate of immune complex degradation by macrophages in vitro, this process was not accompanied by a significantly greater uptake of complexes.

These studies show that impairment of macrophage $\mathrm{Fe}$ release by inflammatory stimuli can be directly demonstrated in vitro, and that the pro-inflammatory cytokine TNF- $\alpha$ appears to play a role in this process, and may do so by a direct effect on macrophage Fe metabolism. This finding is in agreement with other studies indicating an up regulation of ferritin by TNF- $\alpha$ (Miller et al. 1991), which would cause the cell to store more Fe and release less, as originally proposed by Konijn \& Hershko (1977).

It is now known that the activity of IRP, mentioned earlier, is crucial to maintenance of Fe homeostasis in most cells (Haile, 1999), due to its ability to simultaneously and inversely regulate $\mathrm{Fe}$ uptake via the transferrin receptor and $\mathrm{Fe}$ storage by ferritin according to cellular $\mathrm{Fe}$ levels. However, macrophages do not conform completely to the IRP paradigm (Testa et al. 1991), and it is possible that other factors, notably $\gamma$-interferon ( $\gamma$ IFN; Byrd \& Horwitz, 1990), can affect macrophage Fe homeostasis. Since it is known that NO can activate IRP1 independently of Fe status (Weiss et al. 1993; Drapier et al. 1993), it seemed likely that this mediator, whose synthesis is greatly increased when macrophages are stimulated with $\gamma \mathrm{IFN}$, might play a key role, and that modulation of transferrin-bound Fe uptake by macrophages might occur during inflammation.

It has been proposed that $\gamma \mathrm{IFN}$, produced during T-cell or natural killer cell activation, up regulates NO production, thus activating IRP1, which would in turn lead to increased transferrin receptor expression, and thus to an abnormally increased Fe uptake by macrophages (Weiss et al. 1995). While this theory is plausible, and the IRP-activating ability of NO is clearly established, there was little or no data about whether NO actually affected Fe uptake (as opposed to IRP activation). We have, therefore, investigated the role of NO and $\gamma$ IFN on macrophage $\mathrm{Fe}$ uptake and release in vivo (Mulero \& Brock, 1999).

Using the murine macrophage $\mathrm{J} 774$ cell line, it was found that stimulation with $\gamma \mathrm{IFN}$ and LPS caused, as expected, a marked increase in NO production, but surprisingly caused a 
decrease rather than an increase in Fe uptake from transferrin, despite the previously-reported ability of NO to inactivate IRP1. It seemed possible that this decrease might be due to increased release of $\mathrm{Fe}$, an activity previously noted when macrophages are activated to produce NO (Drapier et al. 1991). However, NO did not cause an increase in $\mathrm{Fe}$ release from the macrophages, so the reduction in uptake was presumably a direct effect of NO and/or $\gamma$ IFN and LPS on Fe uptake. This finding contrasted with the ability of NO to activate IRP1 and the fact that we had previously shown that exogenous NO up regulated transferrin receptor mRNA in erythroleukaemia cells (Oria et al. 1995).

Fluorescence-activated cell sorter analysis revealed that $\gamma \mathrm{IFN}$ alone caused a marked decrease in transferrin receptor expression, even in the absence of LPS or NO production, indicating that the decrease in Fe uptake seen in the presence of $\gamma$ IFN and LPS appears to be primarily due to an NO-independent down regulation of transferrin receptor expression by $\gamma \mathrm{IFN}$. The apparent contradiction between this finding and the previously reported up regulation of IRP1 activity by NO was resolved when it was found that although IRP1 was indeed up regulated, activity of IRP2 was decreased by $\gamma$ IFN (Bouton et al. 1998), and that transferrin receptor expression is primarily determined by IRP2 rather than IRP1 activity (Kim \& Ponka, 2000).

Overall, it appears that $\gamma \mathrm{IFN}$ and LPS stimulation of mouse J774 macrophages decreases $\mathrm{Fe}$ uptake from transferrin. The effect of $\gamma$ IFN and LPS may, therefore, be to ameliorate rather than promote the hypoferraemia induced by pro-inflammatory cytokines such as TNF- $\alpha$.

The work described earlier on the effect of NO on macrophage Fe uptake and release was focused towards Fe uptake from transferrin. However, as mentioned earlier, the major source of $\mathrm{Fe}$ uptake by macrophages is probably phagocytosis of effete erythrocytes rather than transferrin, and $\mathrm{Fe}$ acquired by a phagocytic route may be handled differently from transferrin-bound Fe. It was important, therefore, to know whether NO also affected macrophage handling of $\mathrm{Fe}$ acquired by a phagocytic route. We (JH Brock and V Mulero, unpublished results) have, therefore, recently studied the ability of macrophages from iNOS-knockout mice (Wei et al. 1995) to take up and release Fe delivered as transferrin-antitransferrin immune complexes. NO had no effect on the uptake of immune complexes by bone-marrowderived macrophages, but, unlike the situation with Fe taken up from transferrin, release of Fe was clearly enhanced. The mechanism of enhancement remains to be established; it may involve accelerated release of $\mathrm{Fe}$ from the carrier within the phagosome, or enhanced Fe release from the cell itself. However, the overall effect is the same as with $\mathrm{Fe}$ taken up from transferrin, i.e. NO appears to ameliorate rather than potentiate $\mathrm{Fe}$ sequestration in macrophages, and is thus unlikely to promote the hypoferraemia of inflammation.

Clearly, the effect of cytokines and NO on macrophage Fe handling is complex. While pro-inflammatory cytokines such as TNF- $\alpha$ and interleukins 1 and 6 may provoke hypoferraemia, perhaps via up regulation of ferritin, $\gamma \mathrm{IFN}$ and NO appear to work in the opposite direction. It might be speculated that Fe sequestration is of benefit during the early acute stages of infectious disease in order to reduce Fe availability to micro-organisms, but that the subsequent development of an adaptive immune response requires $\mathrm{Fe}$ to be 'unlocked' in order to allow proper immune function; for example, to ensure optimal lymphocyte activation and proliferation.

\section{References}

Alcantara O, Javors M \& Boldt DH (1991) Induction of protein kinase $\mathrm{C}$ mRNA in cultured lymphoblastoid $\mathrm{T}$ cells by irontransferrin but not by soluble iron. Blood 77, 1290-1297.

Alcantara O, Obeid L, Hannun Y \& Boldt DH (1994) Regulation of protein kinase $\mathrm{C}$ (PKC) expression by iron: effect of different iron compounds on PKC- $\alpha$ and PKC- $\beta$ gene expression and the role of the $5^{\prime}$-flanking region of the PKC- $\beta$ gene in response to ferric transferrin. Blood 84, 3510-3517.

Alvarez-Hernández X, Licéaga J, McKay IC \& Brock JH (1989) Induction of hypoferremia and modulation of macrophage iron metabolism by tumor necrosis factor. Laboratory Investigation 61, 319-322.

Baker E \& Morgan EH (1994) Iron transport. In Iron Metabolism in Health and Disease, pp. 63-95 [JH Brock, JW Halliday, MJ Pippard and LW Powell, editors]. London: W.B. Saunders.

Bouton C, Oliveira L \& Drapier JC (1998) Converse modulation of IRP1 and IRP2 by immunological stimuli in murine RAW 264.7 macrophages. Journal of Biological Chemistry 273, 9403-9408.

Brekelmans P, van Soest P, Leenen PJM \& van Ewijk W (1994) Inhibition of proliferation and differentiation during early $\mathrm{T}$ cell development by anti-transferrin receptor antibody. European Journal of Immunology 24, 2896-2902.

Brock JH (1993) Iron and immunity. Journal of Nutritional Immunology 2, 47-106.

Byrd TF \& Horwitz MA (1990) Interferon-gamma activated human monocytes downregulate transferrin receptors and inhibit the intracellular multiplication of Legionella pneumophila by limiting the availability of iron. Journal of Clinical Investigation 83, 1457-1465.

Djeha A \& Brock JH (1992) Effect of transferrin, lactoferrin and chelated iron on human T-lymphocytes. British Journal of Haematology 80, 235-241.

Drapier JC, Hirling H, Wietzerbin J, Kaldy P \& Kuhn LC (1993) Biosynthesis of nitric oxide activates iron regulatory factor in macrophages. EMBO Journal 12, 3643-3649.

Drapier JC, Pellat C \& Henry Y (1991) Generation of EPRdetectable nitrosyl-iron complexes in tumor target cells cocultured with activated macrophages. Journal of Biological Chemistry 266, 10162-10167.

Esparza I \& Brock JH (1981) Release of iron by resident and stimulated mouse peritoneal macrophages following ingestion and digestion of transferrin-antitransferrin immune complexes. British Journal of Haematology 49, 603-614.

Fleming MD \& Andrews NC (1998) Mammalian iron transport: an unexpected link between metal homeostasis and host defense. Journal of Laboratory and Clinical Medicine 132, 464-468.

Freireich EJ, Miller A, Emerson CP \& Ross JF (1957) The effect of inflammation on the utilization of erythrocyte and transferrin bound radioiron for red cell production. Blood 12, 972-983.

Golding S \& Young SP (1995) Iron requirements of human lymphocytes: relative contributions of intra- and extra-cellular iron. Scandinavian Journal of Immunology 41, 229-236.

Haile DJ (1999) Regulation of genes of iron metabolism by the iron-response proteins. American Journal of Medical Science 318, 230-240.

Jurado RL (1997) Iron, infections, and anemia of inflammation. Clinical Infectious Diseases 25, 888-895. 
Kim S \& Ponka P (2000) Effects of interferon-gamma and lipopolysaccharide on macrophage iron metabolism are mediated by nitric oxide-induced degradation of iron regulatory protein 2 . Journal of Biological Chemistry 275, 6220-6226.

Kleber EE, Lynch SR, Skikne B, Torrance JD, Bothwell TH \& Charlton RW (1978) Erythrocyte catabolism in the inflammatory peritoneal monocytes. British Journal of Haematology 39, 41-54.

Konijn AM \& Hershko C (1977) Ferritin synthesis in inflammation. I. Pathogenesis of impaired iron release. British Journal of Haematology 37, 7-16.

Kuvibidila SR, Baliga BS, Warrier RP \& Suskind RM (1997) Iron deficiency reduces the hydrolysis of cell membrane phosphatidyl inositol-4,5-biphosphate during splenic lymphocyte activation in C57BL/6 mice. Journal of Nutrition 128, 1077-1083.

Kuvibidila SR, Kitchens D \& Baliga BS (1999) In vivo and in vitro iron deficiency reduces protein kinase $\mathrm{C}$ activity and translocation in murine splenic and purified $\mathrm{T}$ cells. Journal of Cellular Biochemistry 74, 468-478.

Leberman HM, Cohen A, Lee JWW, Freedman MH \& Gelfand EW (1984) Deferoxamine: a reversible S-phase inhibitor of lymphocyte proliferation. Blood 64, 748-753.

Mainou-Fowler T \& Brock JH (1985) Effect of iron deficiency on the response of mouse lymphocytes to Concanavalin A: The importance of transferrin-bound iron. Immunology 54, 325-332.

Miller LL, Miller SC, Torti SV, Tsuji Y \& Torti FM (1991) Iron-dependent induction of ferritin $\mathrm{H}$-chain by tumor necrosis factor. Proceedings of the National Academy of Sciences USA 88, 4946-4950.

Mulero V \& Brock JH (1999) Regulation of iron metabolism in murine $\mathrm{J} 774$ macrophages: role of nitric oxide-dependent and -independent pathways following activation with gamma interferon and lipopolysaccharide. Blood 94, 2383-2389.
Oria R, Alvarez-Hernández X, Licéaga J \& Brock JH (1988) Uptake and handling of iron from transferrin, lactoferrin and immune complexes by a macrophage cell line. Biochemical Journal 252, 221-225.

Oria R, Sánchez L, Houston T, Hentze MW, Liew FY \& Brock JH (1995) Effect of nitric oxide on expression of transferrin receptor and ferritin, and on cellular iron metabolism in K562 human erythroleukaemia cells. Blood 85, 2962-2966.

Salmerón A, Borroto A, Fresno M, Crumpton MJ, Ley S \& Alarcón B (1995) Transferrin receptor induces tyrosine phosphorylation in $\mathrm{T}$ cells and is physically associated with the TCR $\xi$ chain. Journal of Immunology 154, 1675-1683.

Smith A, Eskew JD, Borza CM, Pendrak M \& Hunt RC (1997) Role of heme-hemopexin in human T-lymphocyte proliferation. Experimental Cell Research 232, 246-254.

Testa U, Kuhn L, Petrini M, Quaranta MT, Pelosi E \& Peschle C (1991) Differential regulation of iron regulatory element-binding protein(s) in cell extracts of activated lymphocytes versus monocytes-macrophages. Journal of Biological Chemistry 266, 13925-13930.

Walter T, Olivares M, Pizarro F \& Muñoz C (1997) Iron, anemia, and infection. Nutrition Reviews 55, 111-124.

Wei XQ, Charles IG, Smith A, Ure J, Feng GJ, Huang FP, Xu D, Muller W, Moncada S \& Liew FY (1995) Altered immune responses in mice lacking inducible nitric oxide synthase. Nature 375, 408-411.

Weiss G, Goossen B, Doppler W, Fuchs D, Pantopoulos K, Werner-Felmayer G, Wachter H \& Hentze MW (1993) Translational regulation via iron-responsive elements by the nitric oxide/NO-synthase pathway. EMBO Journal $\mathbf{1 2}$, 3651-3657.

Weiss G, Wachter H \& Fuchs D (1995) Linkage of cell-mediated immunity to iron metabolism. Immunology Today 16, 495-500. 